\title{
Le médecin, éboueur de la société
}

Ernest Truffer

Correspondance:

Dr Ernest Truffer

Vieilles Cibles 1

CH-3960 Sierre

Tél./fax 0274556771
La démocratie que nous vivons manque décidément de logique et de cohérence. Elle s'oppose d'une part, et au nom des grands principes, à la peine de mort pour les tueurs et les assassins alors que, par ailleurs et sans complexe, elle condamne à cette même peine de mort les innocents dont le seul crime est d'être atteint d'une maladie nécessitant un traitement qu'elle juge financièrement trop élevé. Il y avait jadis les maladies honteuses. Il y a maintenant les maladies coûteuses. Et celles-ci sont devenues mortelles.

Comme l'affirme B. Kiefer de la «Revue médicale suisse» dans «Le Temps» du 9 octobre 2007: «En Suisse, la moitié déjà des morts impliquent une décision (euthanasie passive pour le moins).» Selon l'enquête «European End-ofLife Decisions», rendue publique le 29 janvier 2007 par l'Institut national d'études démographiques (INED) et reprise le lendemain par «Le Monde», il appert qu' «entre un quart et la moitié des décès survenant en Europe feraient l'objet d'une décision médicale susceptible d'abréger la vie des patients». En Suisse, les «décisions médicales avec l'intention explicite de hâter la mort (arrêt des traitements ou administration de substances létales) concernent [...] 21\% [...] des décès» (cité dans [1]). Et nous ne parlons pas d'Exit et de Dignitas!

On croit rêver. Comment la médecine officielle est-elle tombée dans le cloaque éthique actuel? Elle qui, sous la férule de l'Etat et des caisses, détruit les fœtus malades, à risques ou tout simplement gênants, rationne les soins aux patients et tend à les supprimer aux vieillards, sous prétexte qu'ils coûtent trop cher à la collectivité. A-t-elle déjà entendu parler d'Hippocrate et de son serment?

Il est évident que si on en est arrivé là, c'est parce que la médecine hippocratique, fondée sur les besoins propres et prioritaires du malade, s'est dissoute en une médecine fonctionnaire (vétérinaire), dont le but est de soigner le patient en fonction des besoins et des exigences de l'employeur du médecin. Le malade n'est plus qu'une occasion de remplir la mission qu'impose l'Etat au médecin fonctionnaire: réaliser l'option politique de la santé, définie dans sa planification générale. Le cas de chaque malade particulier ne saurait donc entrer en concurrence avec cette option politique.
D'où l'on doit conclure que la conception actuelle de la médecine officielle, sous la botte des caisses parce que payée par celles-ci, est non hippocratique puisqu'elle consiste à soigner le malade «économiquement», c'est-à-dire très exactement comme un paysan le ferait pour son bétail, dont la survie ne relève que des données de rentabilité.

De plus, il faut encore proposer à la méditation du lecteur un phénomène qui n'apparaît, de prime abord, pas nécessairement comme évident. Il s'agit des années de vie dont quantité de citoyens âgés ont été privés, parce que l'ultime chance que pouvait leur offrir la médecine leur a été refusée. Simplement du fait que leur médecin n'a pas osé la saisir, conditionné qu'il est dans son subconscient, depuis des années, par les impératifs de «traitement économique» et culpabilisé par l'explosion des coûts de la santé, dont on l'accuse d'être l'artificier. Alors que justement, grâce aux progrès de l'anesthésiologie et de la pharmacologie, le vieillard peut maintenant bénéficier d'interventions et de thérapies aussi efficaces que celles de l'adulte ou de l'enfant.

Mais la décision du médecin dépendra non seulement de ses connaissances scientifiques mais également et probablement autant, dans les cas limites, de son équilibre psychique, de sa force de caractère. Ce qui signifie qu'il lui faut un psychisme suffisamment solide pour résister à ce conditionnement et ces pressions externes, sous peine de ne pouvoir donner au malade âgé son ultime chance. Car il est plus facile et moins cher, pour l'Etat et les caisses, de le laisser - ou le faire! - mourir.

Aussi, comment, à partir d'un âge certain, faire encore confiance au médecin? Qui garantit qu'il possède une structure psychologique suffisamment indocile pour enfreindre ce conditionnement intensif, ce matraquage continu et malveillant? Si on considère l'évolution actuelle de l'exercice médical, totalement soumis à la dépendance des caisses et de l'Etat alors que, il y a à peine quelques décennies, il était encore indépendant de ces pouvoirs, rien n'est moins certain. Peut-il vraiment résister aux assauts continus des adversaires de la médecine hippocratique et indépendante?

Comment voulez-vous qu'il ne soit pas foncièrement écouré par l'ignoble et incessante 
accusation de "pratiquer l'acharnement thérapeutique pour de sordides raisons financières»? Par la mentalité «d'ayant droit» omniscient, revendicateur et assisté du citoyen cotisant? Par une classe politique, à plat ventre devant le plus puissant lobby du pays, celui des caissesmaladie?

Peut-il être insensible à la désastreuse image de marque que les caisses et l'Etat (ces dits «partenaires» sociaux!) lui ont construite dans l'opinion publique, pour en faire définitivement le «bouc émissaire» de la santé. Est-il en mesure de supporter les pressions constantes que lui font subir les caisses, grâce au «profil médical» et à la maladie statistiquement tarifée que leurs bureaucrates, luxueusement informatisés, ont inventés et mis au point?

Dans le subconscient du médecin, ces théories et ces pressions ne vont-elles pas peser d'un poids décisif sur l'établissement du pronostic (qui décidera du traitement ou du non-traitement), c'est-à-dire sur l'estimation des chances de survie du vieillard, par définition, au soir de sa vie? D'autant plus que, en cas d'échec, la thérapeutique peut économiquement s'alourdir (soins intensifs, réanimation, etc.), détériorant ainsi son «profil médical» et le faire entrer dans la catégorie des médecins dits «chers», empoisonnant ainsi ses rapports futurs, toujours plus contraignants, avec la bureaucratie des caisses.

Il n'y a pas de réponse précise ni générale à ces questions. C'est pourquoi elles doivent être soumises à la réflexion et la méditation du lecteur. Tout en lui faisant remarquer que notre «sécurité» sociale aura ainsi réussi à opposer, dans le cas du vieillard (pour l'instant!), la chance vitale (sa survie) du malade au risque socioéconomique du médecin.

Voilà une situation méritant réflexion que les media n'ont pas l'habitude de proposer ...

\section{Référence}

1 Une enquête sur la fin de vie en Europe. Oui à la vie. 2007;37 (Mars):4. 